\title{
COMPORTAMIENTO AGRONÓMICO DE CULTIVARES DE MELÓN (Cucumis melo L. var. Inodorus) BAJO INVERNADERO EN LA ZONA ESTE DEL CHACO
}

\section{Agronomical performance of melón cultivars (Cucumis melo L. var. Inodorus) un- der greenhouse conditions in the east zone of Chaco}

\author{
Monteros Solito, Ramiro I.; Avico, Eda L.; Shindoi, Mauro M. F. J. y Sarco, Pamela C. \\ INTA. Producción Vegetal y Gestión Ambiental-EEA Colonia Benítez, Chaco, Argentina. \\ e- mail: monterosolito.ramiro@inta.gob.ar
}

\begin{abstract}
RESUMEN
El objetivo de este trabajo fue evaluar el comportamiento de tres cultivares de melón tipo Orange Honey Dew bajo invernadero. Estos fueron cv Silver 1, cv Silver 2 y cv Silver 3 de American Seeds ${ }^{\circledR}$. Se trasplantó a los 30 días después de la siembra cuando alcanzaron a tener tres hojas verdaderas. La conducción del cultivo fue vertical y sin poda de las guías. Se registraron los días posteriores de trasplante del comienzo y fin de cosecha por cultivar. A cosecha se midió: $\mathrm{kg} \mathrm{planta}^{-1}$, peso promedio de frutos, número de frutos planta ${ }^{-1}$, Sólidos Solubles Totales y diámetro de los frutos; se calculó el rendimiento total en tn.ha ${ }^{-1}$. El período de trasplante a cosecha para los tres cultivares fue de 128 días desde el trasplante. No hubo diferencias significativas entre los cultivares en las variables estudiadas. Todos los cultivares registraron pesos mínimos del fruto por arriba de $0,6 \mathrm{~kg}$ considerados comerciales y el rendimiento estuvo en el rango de 55,3-66 tn ha ${ }^{-1}$. Por lo que el cultivo de melón bajo invernadero ofrece una alternativa para el sistema de hortícola diversificado de la zona del Este del Chaco.
\end{abstract}

Palabras clave: rendimiento melón, hortalizas, sistema intensivo

\begin{abstract}
The aim of this work was to evaluate the performance of three Orange Honey Dew melon cultivars in a greenhouse. These were cv Silver 1, cv Silver 2 and cv Silver 3 from American Seeds ${ }^{\circledR}$. They were transplanted 30 days after sowing when they reached three true leaves. The crop was trained vertically without pruning the guides. After transplanting, the beginning and ending of the harvest of each cultivar was recorded. During the harvest, $\mathrm{kg}_{\text {plant }}{ }^{-1}$, average fruit weight, number of fruits per plant ${ }^{-1}$, Total Soluble Solids, and fruit diameter were measured; the total yield was calculated in tn.ha'. The period between the transplant and the harvest was 128 days long for the 3 cultivars. There were no significant differences among the cultivars in the variables studied. All cultivars recorded minimum fruit weight above $0,6 \mathrm{~kg}$ which is fesasible for commerce and yield was within the range of 55,3-66 tn ha ${ }^{-1}$. Therefore, melon crops in greenhouses offer a plausible alternative for the diversified horticultural system in the Eastern Chaco area.
\end{abstract}

Key words: melon yield, vegetables, intensive system

Recibido: 22/Jun/2021. Aceptado: 20/Ago/2021 


\section{INTRODUCCIÓN}

Los cinturones verdes o cinturones hortícolas se han desarrollado alrededor de las principales ciudades y se caracterizan por la producción de alimentos frescos (frutas y hortalizas) para la población urbana (Titonell y Giobellina, 2018). El cinturón hortícola del Gran Resistencia se caracteriza por la cercanía de la población urbana demandante de productos inocuos y la continua expansión de la urbe sobre tierras productivas (Alberto et al., 2018). En ese sentido hay que buscar estrategias para intensificar la producción hortícola dentro de esta interfase urbano-rural, campo-ciudad o rururbano, con especial énfasis en el cuidado del ambiente, la diversificación y el manejo eficiente de los recursos disponibles (Barsky, 2005) Un estudio realizado en el Este del Chaco por Sarco et al. (2018), mostró como segundo cultivo en importancia, detrás de las hortalizas de hojas a las cucurbitáceas. El cultivo de Cucumis melo var. Inodorus con tajada es el más difundido (melón criollo con tajada) (García y Bravo 2015) y se realiza a campo, con escasa incorporación de tecnología. La producción bajo invernadero es una alternativa que presenta varias ventajas sobre el cultivo a campo abierto, permite la oferta fuera de estación en épocas de alta demanda, mayor número de cosechas durante el año, precocidad de la cosecha, economía de agua y de fertilizantes, mayor rendimiento y disminución de problemas fitosanitarios con la consecuente pérdida de producción, mejorando así la calidad de los frutos (Vargas et al. 2008; Szczesny et al., 2014). Esto se logra mediante la implementación de una práctica importante que es el uso del tutorado (Gomez-Guillamón et al. 1997). Esta técnica pretende mejorar la administración de la superficie y del espacio cultivado con el objetivo de mejorar la densidad de plantación y en consecuencia el rendimiento por superficie (FAO, 2002; Reche, 2007).

Por esto, es necesario generar información sobre el comportamiento del cultivo protegido de melón, como una alternativa para el sistema hortícola diversificado de la zona del Este del Chaco.

\section{Objetivo}

Evaluar el comportamiento agronómico bajo invernadero de tres cultivares de melón para la zona Este del Chaco.

\section{MATERIALES Y MÉTODOS}

El ensayo se llevó a cabo en el invernadero del módulo experimental frutihortícola de la EEA INTA Colonia Benítez-Chaco, tiene una medida de $5 \mathrm{~m}$ de ancho por $20 \mathrm{~m}$ de largo, de estructura metálica y techumbre curva sin abertura cenital.

Los materiales usados para la evaluación agronómica fueron híbridos del tipo Orange Honey Dew cv Silver 1 , cv Silver 2 y cv Silver 3 de American Seeds ${ }^{\circledR}$. Se sembró el 3/08/2019 en speedling de 72 celdas. Se trasplanto a una densidad de 2,56 plantas. $\mathrm{m}^{-2}$ (Monge-Pérez y Loría-Coto, 2017) $(0,3 \mathrm{~m}$ entre plantas y $1,2 \mathrm{~m}$ entre líneos) el 02/09/2019 cuando alcanzaron a tener 3 hojas verdaderas (Figura 1a).

Se realizó el cálculo de necesidades nutricionales para un rendimiento potencial de $70 \mathrm{tn} \mathrm{ha}^{-1}$, con una extracción por tn de $3,5 \mathrm{~kg}$ de N, $1,05 \mathrm{~kg}$ de $\mathrm{P}_{2} \mathrm{O}_{5}$ y $7 \mathrm{~kg}$ de $\mathrm{K}_{2} \mathrm{O}$ (Sánchez et al., 1998).

La conducción del cultivo fue vertical, sin poda de guías, tutorándose las guías con hilo de polipropileno (Figura 1b).

El diseño experimental del ensayo fue en bloques al azar con tres repeticiones. Se cosecharon ocho plantas centrales del lineo por cultivar y repetición. Se determinó inicio de cosecha en función de la apariencia externa de los frutos determinado por anillo amarillo en la unión peduncular, coloración crema, sin brillo y ausencia de pilosidad (Mármol, 2008; Díaz y Monge-Pérez, 2019). Se registraron los días posteriores de trasplante a floración, comienzo y fin de cosecha por cultivar.

A cosecha se evaluaron por cultivar: $\mathrm{kg} \mathrm{planta}^{-1}$, peso promedio de frutos, número de frutos planta ${ }^{-1}$, Sólidos Solubles Totales (SST) utilizando un brixómetro y diámetro de los frutos. Se calculó el rendimiento total en tn $\mathrm{ha}^{-1}$. Los datos fueron sometidos al ANOVA con prueba de Duncan utilizando el software Infostat.

\section{RESULTADOS Y DISCUSIÓN}

El ciclo del cultivo de trasplante a floración fue de 53 días (Figura 3 a) y a cosecha fue de 81 días posteriores al trasplante -DPT-, para los tres cultivares. La cosecha se inició el 22/11/2019 (Figura 2 a) y finalizó el 08/01/2020 completando el ciclo en 128 DPT. El momento de máxima cosecha se da entre los 94 y 117 DPT que coincide con los días 03 y 26/12/2019 (Figura 3). 
a)

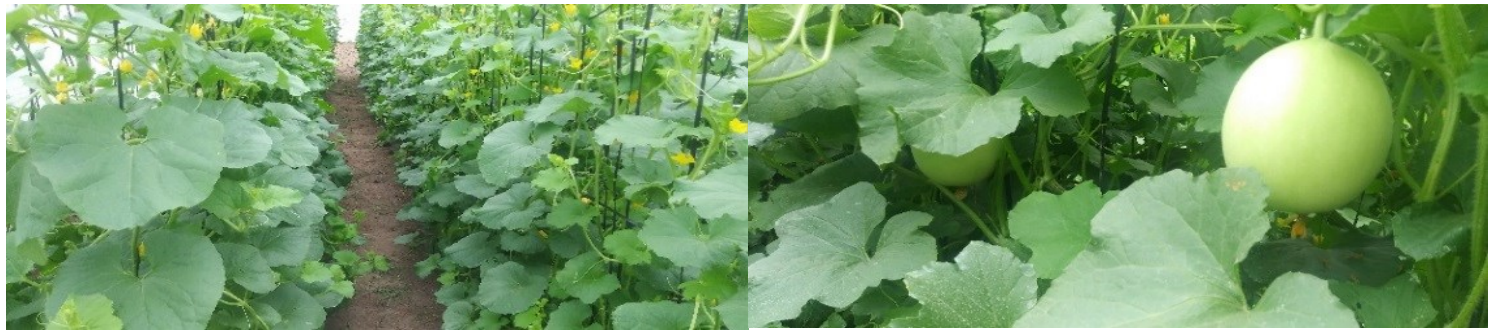

b)

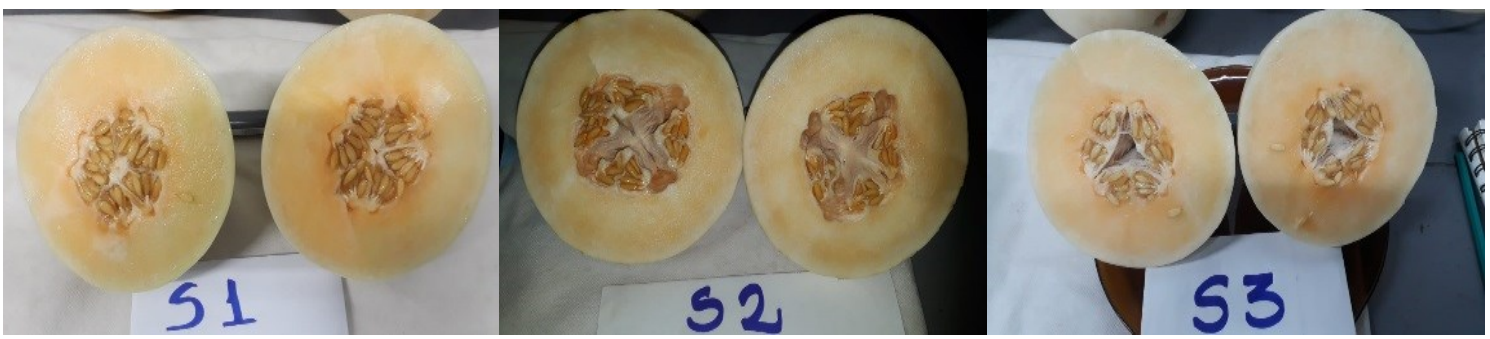

Figura 2. a) Plantas en plena floración y fructificación b) Aspecto interno de los diferentes cultivares.

El fruto de melón a diferencia que los que se producen a campo en la zona de estudio presenta pulpa color naranja (figura 2 b), es el caso de los tres cultivares evaluados con característica de tipo Orange Honey Dew.

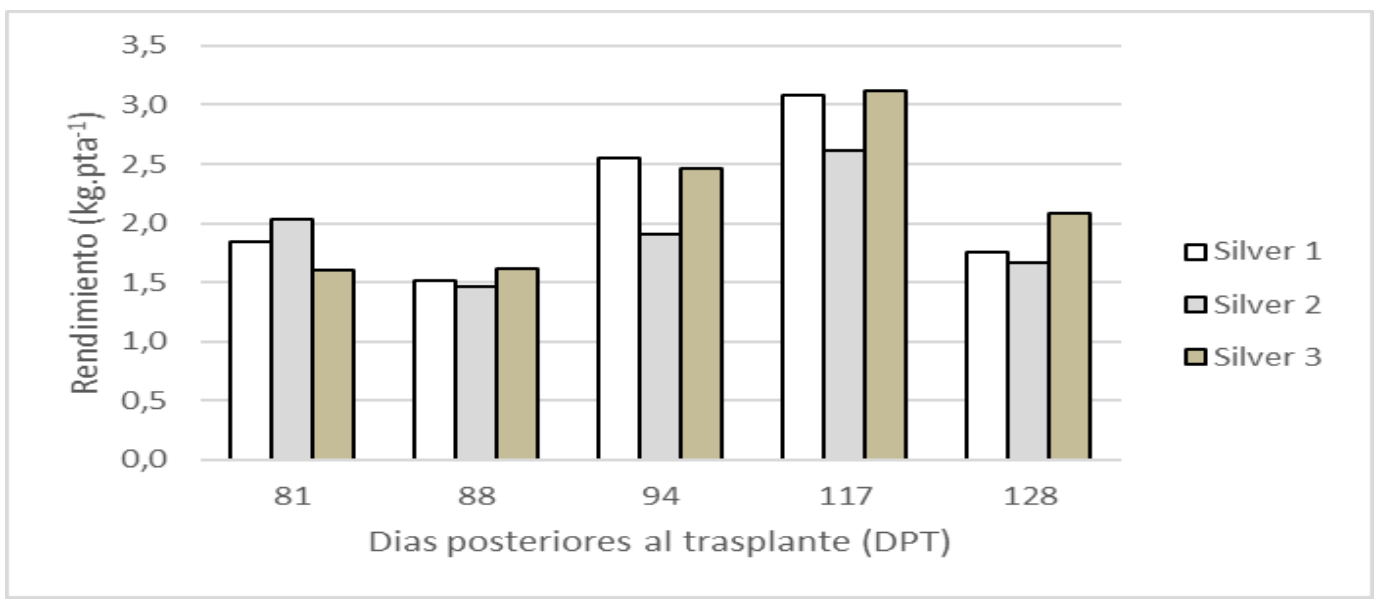

Figura 3. Rendimiento promedio por cosecha y cultivar en $\mathrm{kg} \mathrm{planta}^{-1}$

El análisis de los kilogramos de frutos obtenidos por planta entre cultivares (Tabla 1) no evidenció diferencias significativas, estos valores estuvieron entre $2,16-2,58 \mathrm{~kg}$, por encima de los 1,89 $\mathrm{kg}$ hallados por Monge Pérez y Loría Coto (2017).

Los pesos promedios de los frutos estuvieron en el rango de 1,39-1,59 kg, sin diferencias significativas entre cultivares y por encima de los hallados en otros trabajos para híbridos del tipo Honey Dew, 0,837 kg (Monge Pérez y Loría-Coto, 2017); 0,825 kg (Monge Pérez, 2016); 0,97-1,12 kg (Díaz, 2013) y por debajo de los 2,02 kg obtenidos por Bezerra et al. (2009).

La cantidad de frutos por planta estuvo entre 1,60-1,80, sin diferencias significativas entre cultivares. Los valores de rendimiento por planta hallados en este ensayo, estuvieron por encima de los registrados por otros autores, 1,38 kg planta ${ }^{-1}$ (Monge-Pérez, 2016); y el rango de 0,70-1,30 kg planta ${ }^{-1}$ (Díaz, 2013); 0,60-1,00 kg planta $^{-1}$ (Botto, 2011) y por debajo de los 2,21 kg planta ${ }^{-1}$ (Monge-Pérez y Loría-Coto, 2017).

Para el diámetro obtenido de los frutos tampoco se observaron diferencias entre cultivares. En la variable SST no se hallaron diferencias entre cultivares. Por otra parte, como se evidenció en la tabla 1, Todos los cultivares registraron pesos mínimos de fruto superiores a $0,6 \mathrm{~kg}$. 
El rendimiento total estuvo entre 55,3 y $66 \mathrm{tn} \mathrm{ha}^{-1}$, superiores a los encontrados por otros autores, 21-30 tn ha $^{-1}$ (Bezerra et al. 2009); 47,5 tn ha ${ }^{-1}$ (Monge-Pérez y Loría-Coto, 2017) y 49,5 tn ha ${ }^{-1}$ (Díaz, 2013) .

Tabla 1. Resumen de las variables medidas y de rendimiento según cultivar. Medias \pm D.E. con una letra común no son significativamente diferentes $(\mathrm{p}>0,05)$

\begin{tabular}{|c|c|c|c|}
\hline Variables & cv Silver 1 & cv Silver 2 & cv Silver 3 \\
\hline $\mathrm{kg}$ de fruto planta ${ }^{-1}$ & $2,58 \pm 1,76 \mathrm{a}$ & $2,16 \pm 1,37 \mathrm{a}$ & $2,55 \pm 1,48 \mathrm{a}$ \\
\hline Frutos planta $^{-1}$ & $1,80 \pm 1,04 \mathrm{a}$ & $1,60 \pm 1,01 \mathrm{a}$ & $1,64 \pm 0,85 \mathrm{a}$ \\
\hline Peso del fruto (kg) & Peso del fruto (kg) & Peso del fruto $(\mathrm{kg})$ & Peso del fruto (kg) \\
\hline Promedio & $1,45 \pm 0,45 \mathrm{a}$ & $1,39 \pm 0,37 \mathrm{a}$ & $1,59 \pm 0,51 \mathrm{a}$ \\
\hline Mínimo & 0,63 & 0,66 & 0,76 \\
\hline Máximo & 2,38 & 2,05 & 2,54 \\
\hline Diámetro del fruto $(\mathrm{cm})$ & $9,78 \pm 1,02 \mathrm{a}$ & $9,55 \pm 1,56 \mathrm{a}$ & $10,47 \pm 1,66 \mathrm{a}$ \\
\hline SST ( ${ }^{\circ}$ Brix) & $9,67 \pm 2,08 \mathrm{a}$ & $12,78 \pm 2,13 \mathrm{a}$ & $11,17 \pm 3,01 \mathrm{a}$ \\
\hline Rendimiento (tn $\left.\mathbf{h a}^{-1}\right)$ & $66 \pm 44,9 \mathrm{a}$ & $55,3 \pm 34,96 a$ & $65,3 \pm 37,8 \mathrm{a}$ \\
\hline
\end{tabular}

\section{CONCLUSIÓN}

Los cultivares cv Silver 1, cv Silver 2 y cv Silver 3 de melón tipo Orange Honey Dew, producidos bajo invernadero con conducción vertical y sin poda de guías evaluados en este trabajo, ofrecen una alternativa viable al sistema productivo hortícola de la zona del Este del Chaco. La posible incorporación de estos cultivares de pulpa naranja a los sistemas productivos de altos rendimientos, buena calidad de fruto y con una oferta oportuna en el momento de alta demanda, podría resultar una interesante opción para el productor.

\section{REFERENCIAS}

Alberto, J.A., Mignone, A., Arce, G.A. y López, S. (2018). Dinámica y desarrollo urbano del área metropolitana del Gran Resistencia: organización espacial en las primeras décadas del S. XX. Contribuciones Científicas GAEA, 30: 30-35.

Barsky, A. (2005). "El periurbano productivo, un espacio en constante transformación. Introducción al estado del debate, con referencias al caso de Buenos Aires". Scripta Nova, 9 (194): 36, Barcelona.

Bezerra, F.M., Nunes, M.C.; Freitas, C.A. y Silva, F.L. (2009). Desempenho de três híbridos de meloeiro sob dois espaçamentos em ambiente protegido na Chapada do Apodi. Rev. Ciênc. Agron., Fortaleza, 40 (3): 412-416.

Botto, A.S. (2011). Evaluación del rendimiento y el total de sacarosa disuelta $\left({ }^{\circ} \mathrm{Bx}\right)$ de quince cul-tivares de melón (Cucumis melo L.) en sustrato compost y mezcla compost con arena bajo condi-ciones de macrotúnel. Zamorano, Honduras: Escuela Agrícola Panamericana.

Díaz, A.J.M. (2013). Efecto de la poda y la densidad de siembra sobre el rendimiento y la calidad de fruta de melón (Cucumis melo L.) cultivado en invernadero. San Pedro de Montes de Oca, San José, Costa Rica: Universidad de Costa Rica.

Díaz, A.J.M. y Monge Pérez, J.E. (2019). Evaluación económica del uso de diversos tipos de poda y densidades de siembra en melón tipo Honey dew (Cucumis melo L.) cultivado en invernadero. La investigación en Guanacaste III. 115-135 p. San José, Costa Rica: Ed. Nuevas Perspectivas.

FAO. (2002). El cultivo protegido en clima mediterráneo. Roma, Italia. Organización de las Naciones Unidas para la Agricultura y la Alimentación. 
García, P. y Bravo, S. (2015). Conocimiento y conservación de semillas agrícolas originarias cultivadas por familias campesinas en el Salado Centro, Santiago del Estero. Memorias del V Congreso Latinoamericano de Agroecología. Archivo Digital: descarga y online ISBN 978-950-34-1265-7.

Gómez-Guillamón, M.L., Camero, R. y González-Fernández, J. (1997). El melón en invernadero. En A. Namesny, Melones. 67-77 p. Barcelona, España: Ed. De Horticultura S. L.

Mármol, J.R. (2008). Cultivo de melón en invernadero. Edita: Junta de Andalucía, Consejería de Agricultura y Pesca. 312 pp.

Monge-Pérez, J.E. (2016). Evaluación de 70 genotipos de melón (Cucumis melo L.) Cultivados bajo invernadero en Costa Rica. Rev. Intersedes. 17 (36). DOI: http://dx.doi.org/10.15517/isucr.v17i36.26944

Monge-Pérez, J.E. y Loria Coto, M. (2017). Producción de melón en invernadero: comparación agronómica entre tipos de melón. Rev. Posgrado y Sociedad Sistema de Estudios de Posgrado Universidad Estatal a Distancia 15 (2): 79-100. https://doi.org/10.22458/rpys.v15i2.1966

Obregón, V. (2017). Guía para la identificación de las enfermedades de las cucurbitáceas. Ediciones INTA. Libro digital PDF. Archivo Digital: descarga y online. https://inta.gob.ar/sites/default/files/inta_guia identificacion de las enfermedades de las cucurbitaceas.pdf

Reche, J. (2007). Cultivo intensivo del melón. Madrid. España: Ministerio de Agricultura, Pesca y Alimentación.

Sánchez, L.R., Sironi, J.S., Crespo, J.A.P., Pellicer, C. y López, M.D.G. (1998). Crecimiento y absorción de nutrientes del melón bajo invernadero. Investigación Agraria Producción Protección Vegetales, Madrid, 13 (1-2):111-120.

Sarco, P., Avico, E., Monteros, D., Vagabculob, J., Vagabculob, J., Francescutti, F.; Lestani, M.; y Shindoi, M. (2018). Diagnóstico de la actividad hortícola en la zona de influencia de la EEA INTA Colonia Benítez, Chaco.

Szczesny, A.E., Adlercreutz, D., Huarte, A., López Camelo, E., Manzo y Viglianchino, L. (2014). Producción hortícola bajo cubierta 150 p. Instituto Nacional de Tecnología Agropecuaria (INTA), Ciudad Autónoma de Buenos Aires, Argentina. https://inta.gob.ar/sites/default/files/script-tmp-inta-_prod_hort_bc.pdf

Tittonell, P. y Giobellina, B. (2018). PERIURBANO hacia el consenso: ciudad, ambiente y producción de alimentos: propuestas para ordenar el territorio: resúmenes ampliados: libro $11^{\text {a }}$ Ed.-Ciudad Autónoma de Buenos Aires: Ediciones INTA. Libro digital, PDF Archivo Digital: https://inta.gob.ar/sites/default/files/ inta_periurbanos_hacia_el_concenso_libro_2_resumenes_cortos.pdf

Vargas, P.F., Castoldi, $\bar{R}$., Chärlo, H.C. y Braz, $\bar{L} . \bar{T}$. (2008). Qualidade de melão ren-dilhado (Cucumis melo L.) em função do sistema de cultivo. Ciência e Agrotecnología, 32 (1): 137-142. https://doi.org/10.1590/ S1413-70542008000100020 